\title{
Comparación de Helicobacter pylori en cavidad oral y mucosa gástrica de acuerdo a genotipo de virulencia ( $\operatorname{cag} A$ y vacAm 1)
}

\author{
Ester Sepúlveda, Jessica Moreno, María L. Spencer, Sandra Quilodrán, \\ Ursula Brethauer, Carlos Briceño y Apolinaria García
}

\section{Universidad de Concepción, Concepción, Chile. Facultad de Odontología Departamento de Patología y Diagnóstico (ES, MLS, UB). Facultad de Ciencias Biológicas Departamento de Microbiología (SQ, AG). \\ Facultad de Medicina} Departamento de Medicina Interna

Universidad Andrés Bello, Concepción, Chile. Facultad de Ciencias Biológicas Departamento de Ciencias Biológicas (JM).

Trabajo realizado en el Laboratorio de Patogenicidad Bacteriana, Departamento de Microbiología, Facultad de Ciencias Biológicas,

Universidad de Concepción.

Fuente de financiamiento: Proyecto DIUC N²06.103.017-1.0, proyecto Fondef N N03i1105.

Los autores declaran no tene conflictos de interés

Recibido: 30 de agosto de 2011 Aceptado: 29 de febrero de 2012

Correspondencia a: Ester Sepúlveda Tebache esepulve@udec.cl

\section{Comparison of Helicobacter pylori in oral cavity and gastric mucosa according to virulence genotype ( $\operatorname{cag} A$ and $\operatorname{vac} A \mathrm{~m} 1)$}

Objective: To compare the virulence genotype (cagA and vacA $\mathrm{m} 1$ genes) of Helicobacter pylori obtained simultaneously from gastric mucosa and oral cavity. Material and Methods: Gastric samples of 18 patients were obtained by endoscopic biopsies. Oral samples of these patients were obtained from dental plaque and saliva swabs from the floor of the mouth and the base of the tongue. All samples were studied by conventional PCR and real-time PCR (RT-PCR). Virulence genes $\operatorname{cag} A$ and $v a c A \mathrm{~m} 1$ were studied by RT- PCR. Results: According to presence and/or absence of $\operatorname{cag} A$ and $v a c A m 1$ genes, seven different combinations were observed. Conclusion: These results suggest that there is a variety of genetic profiles of Helicobacter pylori in the stomach and oral cavity, with a predominance of less virulent genotypes in the patients included in this study (cagA-, vacA m1-).

Key words: Helicobacter pylori, virulence genes, real-time PCR.

Palabras clave: Helicobacter pylori, genes de virulencia, RPC en tiempo real.

\section{Introducción}

H elicobacter pylori ha sido asociado a una variedad de patologías gastro-duodenales, especialmente carcinoma gástrico ${ }^{1}$. En Chile, el carcinoma gástrico es la principal causa de muerte en hombres y la tercera en mujeres ${ }^{2}$. La prevalencia de $H$. pylori en Chile varía de 73 a $83 \%$, dependiendo del área geográfica $^{3,4}$. Un estudio reciente ${ }^{5}$ realizado en niños entre 3 y 14 años de Santiago de Chile, demostró una prevalencia de $26 \%$, con mayor proporción en los varones.

Helicobacter pylori posee una variedad de factores de virulencia como flagelo, morfología espiralada, adhesinas, enzimas y lipopolisacáridos ${ }^{6,7}$. Destacan entre sus factores de virulencia las proteínas CagA y VacA, las cuales pueden ser investigadas más fácilmente a través de los genes que las codifican, que se denominan genes asociados a virulencia, específicamente en este caso, $\operatorname{cag} A$ y $v a c A^{8-11}$. Cabe destacar que los genes $c a g A$ y $v a c A$ son actualmente importantes marcadores genéticos asociados a virulencia en $H$. pylori ${ }^{12}$.

El gen $\operatorname{cag} A$ codifica la síntesis de la proteína CagA. La producción de esta proteína se potencia por la presencia de pH ácido y es mediada por un sistema de secreción tipo IV. La proteína CagA induce a nivel de las células epiteliales gástricas la producción de citoquinas inflamatorias, como la IL-8, y el reclutamiento de leucocitos. El efecto directo sobre las células gástricas es la reducción de la secreción de ácido ${ }^{13}$. Por otra parte, el gen vacA codifica la producción de toxina $\mathrm{VacA}$, la que presenta un mosaico genético basado en variaciones de alelos en las regiones medias (alelos $m 1$ o $m 2$ y subtipos) y variaciones de señalización

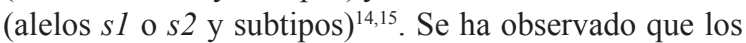
tipos $s 1 / m 1$ son más agresivos y son llamados $\operatorname{Tox}^{+16}$. La toxina VacA es secretada por aproximadamente $50 \%$ de los tipos de $H$. pylori, causando degeneración vacuolar de las células epiteliales gástricas y ulceración de la mu$\operatorname{cosa}^{17}$. Se ha demostrado que los tipos vacA s $1 / \mathrm{m} 1$ tienen una actividad citotóxica aumentada en comparación con los tipos $s 1 / m 2$ y que los tipos $s 2 / m 2$ no poseen ninguna actividad citotóxica ${ }^{14}$.

Estudios realizados por nuestro grupo ${ }^{18,19}$, incluyendo muestras de biopsias gástricas de pacientes de diversas regiones de Chile, señalan la importancia de los genes cagA y vacA m1 en la patogenicidad de $H$. pylori, razón por la cual fueron considerados en esta investigación. Cabe destacar que los resultados de dichas investigaciones generaron el consecuente desarrollo de un kit molecular en proceso de patentamiento ("Un kit molecular de diagnóstico de cepas virulentas de Helicobacter pylori", patente en trámite, número de solicitud: 1520-08).

Diferentes investigaciones demuestran la presencia de H. pylori en la cavidad oral (placa bacteriana y saliva), actuando como un reservorio para la bacteria ${ }^{20-25}$. Algunos autores han encontrado una asociación entre la presencia de $H$. pylori en mucosa gástrica y oral ${ }^{26-33}$. En una investigación previa realizada por nuestro grupo, se observó la co-existencia de la bacteria en ambas localizaciones mediante RPC en tiempo real (RPC-TR) ${ }^{34}$. En este estudio se observó que de 54 pacientes, $39 \%(n=21)$ 
presentó muestras gástricas positivas para $H$. pylori. Todas las muestras orales de estos 21 pacientes resultaron negativas para $H$. pylori mediante cultivo. Sólo una muestra de placa bacteriana fue positiva mediante RPC convencional y todas las muestras de saliva resultaron negativas. Sin embargo, las muestras orales de todos los pacientes resultaron positivas mediante RPC-TR (20/21 placa bacteriana, 21/21 saliva del piso de la boca y 20/21 saliva de la base de la lengua).

Se ha estudiado la presencia de genes de virulencia de H. pylori proveniente de estómago y cavidad oral del mismo paciente ${ }^{35,36}$, pero de acuerdo a nuestro conocimiento, no existen investigaciones en la población chilena. Por lo anterior, el objetivo del presente estudio fue comparar los genotipos asociados a virulencia ( $\operatorname{cag} A$ y $v a c A \mathrm{~m} 1) \mathrm{de}$ $H$. pylori obtenido simultáneamente de mucosa gástrica y cavidad oral a partir del mismo paciente.

\section{Material y Métodos}

Pacientes. Se estudiaron muestras gástricas y orales de 21 pacientes con indicación médica de endoscopia digestiva alta, de la Unidad de Gastroenterología del Hospital Regional de Concepción. Se excluyeron pacientes con tratamiento antimicrobiano para $H$. pylori y se obtuvo un consentimiento informado de cada paciente. Esta investigación fue aprobada por la Dirección de Investigación de la Universidad de Concepción.

Obtención de muestras. Las muestras gástricas y orales fueron obtenidas de cada paciente al momento de realizar la endoscopia digestiva. Las muestras orales se obtuvieron de placa bacteriana sub-gingival, con una sonda dental, y las muestras de saliva fueron obtenidas del piso de boca y base de lengua con tórulas de algodón estériles. Las biopsias gástricas se tomaron del antro y cuerpo durante la inspección endoscópica del estómago.

Aislamiento de ADN. El ADN del control positivo de H. pylori ATCC se obtuvo con el kit E.Z.N.A. ${ }^{\circledR}$ para ADN bacteriano, siguiendo las instrucciones del fabricante (OMEGA bio-tek, Norcross, GA, USA). Para las muestras orales se utilizó el kit BIO 101 Protocol Fast DNA E.Z.N.A. ${ }^{\circledR}$ (OMEGA bio-tek, Norcross, GA, USA) y para las muestras gástricas se utilizó el kit E.Z.N.A. ${ }^{\circledR}$ para ADN tisular (OMEGA bio-tek, Norcross, GA, USA), siguiendo las instrucciones del fabricante.

Diseño y elección de Oligos. Se obtuvo por lo menos 15 pares de partidores y sondas mediante el sotfware Oligo 6.2, para la detección de $\beta$-actina (específica para ADN humano), para la región $16 \mathrm{~S}$ ARNr de $H$. pylori y para los genes de virulencia cagA y vacA $\mathrm{m} 1$, eligiendo finalmente sólo un par de ellos, usando los códigos de acceso de sus secuencias mostrados en la Tabla 1. La herramienta BLASTn (Basic Local nucleotid Alignment Search Tool), disponible en NCBI (National Center Biotechnology), permitió encontrar regiones con similitud de secuencias nucleótido por nucleótido. Los criterios de la selección de los partidores fueron los que tenían las mejores condiciones termodinámicas arrojadas por el programa Oligo 6.2 para el de $\beta$-actina y que además coincidieran con alguno ya probado por otros autores, tanto para el gen $16 \mathrm{~S} \mathrm{ARNr}^{37}, c a g A^{38}$ y vacA m1 $1^{39}$. Los porcentajes de identidad fueron de $100 \%$ para los oligos elegidos, dando un mayor puntaje de probabilidad de unión. En la Tabla 1 se detallan los partidores elegidos, los productos esperados y su código NCBI.

Tabla 1. Detección de Helicobacter pylori. Secuencias de partidores y sondas de la amplificación de las zonas de los genes 165 ARNr, cagA, vacA $m 1$ y $\beta$-actina

\begin{tabular}{|c|c|c|}
\hline Gen & Secuencia del partidor $\left(5^{\prime}-3^{\prime}\right)$ & $\begin{array}{l}\text { Tamaño y localización del producto de } \\
\text { amplificación }\end{array}$ \\
\hline $\begin{array}{l}\text { 16S ARNra } \\
\text { Partidor sentido } \\
\text { Partidor antisentido } \\
\text { Sonda }\end{array}$ & $\begin{array}{l}\text { TGC GAA GTG GAG CCA ATC TT } \\
\text { GGA ACG TAT TCA CCG CAA CA } \\
\text { (FAM) CCT CTC AGT TCG GAT TGT AGG CTG CAA C (TAMRA) }\end{array}$ & $\begin{array}{r}119 \mathrm{bp}(1381-1499) \\
(1408-1435)\end{array}$ \\
\hline $\begin{array}{l}\operatorname{cag}^{\mathrm{b}} \\
\text { Partidor sentido } \\
\text { Partidor antisentido }\end{array}$ & $\begin{array}{l}\text { ATA ATG CTA AAT TAG ACA ACT TGA GCG A } \\
\text { TTA GAA TAA TCA ACA AAC ATC ACG CCA T }\end{array}$ & 298 bp (1217-1515) \\
\hline $\begin{array}{l}\text { vacA } \mathrm{m}^{\mathrm{c}} \\
\text { Partidor sentido } \\
\text { Partidor antisentido }\end{array}$ & $\begin{array}{l}\text { GGT CAA AAT GCG GTC ATG G } \\
\text { CCA TTG GTA CCT GTA GAA AC }\end{array}$ & 290 bp (2741-3030) \\
\hline $\begin{array}{l}\beta \text {-actina }^{d} \\
\text { Partidor sentido } \\
\text { Partidor antisentido }\end{array}$ & $\begin{array}{l}\text { CGG AAC CGC TCA TTG CC } \\
\text { ACC CAC ACT GTG CCC ATC TA }\end{array}$ & 289 bp (552-841) \\
\hline
\end{tabular}


$R P C$. Las muestras gástricas y orales fueron ratificadas con RPC convencional y RPC-TR mediante la amplificación de una región del gen $16 \mathrm{~S}$ ADNr. Los genes de virulencia $\operatorname{cag} A$ y vacA $\mathrm{m} 1$ fueron estudiados con RPCTR. Las muestras de 3 pacientes fueron descartadas, ya que no existía suficiente muestra gástrica, dejando un total de 18 pacientes. Para confirmar la amplificación del ADN, como control interno, se investigó la presencia del gen de la $\beta$-actina mediante RPC-TR. Como control negativo se utilizó ADN de Lactobacillus spp., agua grado RPC como blanco y el control positivo ADN de H. pylori ATCC 43504.

Mezcla para RPC convencional. El preparado para RPC consistió en $10 \mathrm{ml}$ de ADN, 2,5 ml de solución tampón (10X), 0,5 $\mathrm{ml}$ de dNTPs $(10 \mathrm{mM}), 1,25 \mathrm{ml}$ de $\mathrm{MgCl}_{2}(50 \mathrm{mM}), 0,125 \mathrm{ml}$ de ADN Taq polimerasa, $\mathrm{H}_{2} \mathrm{O}$ grado RPC para un volumen total de $25 \mathrm{ml}$, y 1,25 ml de partidores sentido y antisentido, que se muestran en la Tabla 1. Los parámetros para RPC convencional consistieron en un período de pre-incubación inicial de 15 min a $95^{\circ} \mathrm{C}$, un período de denaturación de $30 \mathrm{~s}$ a $95^{\circ} \mathrm{C}$, seguido de 45 ciclos de alineamiento a $55^{\circ} \mathrm{C}$ por $30 \mathrm{~s}$, un período de extensión a $72^{\circ} \mathrm{C}$ por $2 \mathrm{~min}$ y luego por $5 \mathrm{~min}$, y finalmente enfriamiento a $4^{\circ} \mathrm{C}$. Los productos $\mathrm{RPC}$ se visualizaron mediante electroforesis en gel de agarosa al $1,8 \%$ bajo luz UV.

Mezcla para RPC-TR. Detección de H. pylori $16 \mathrm{~S}$ ARNr mediante RPC-TR con sonda de hidrólisis TaqMan. Se siguieron las instrucciones del fabricante para la mezcla de amplificación lista para usar LightCycler ${ }^{\circledR}$ TaqMan $^{\circledR}$ Master, para el equipo de LightCycler 2.0 de Roche (catálogo 04735536001), que consiste en 9,1 $\mu$ l de $\mathrm{H}_{2} \mathrm{O}$ grado RPC, $0,5 \mu \mathrm{L}$ de $10 \mu \mathrm{M}$ de sonda TaqMan, $1,4 \mu \mathrm{L}$ de mezcla de partidores $16 \mathrm{~S}$ ARNr de concentración 10 $\mu \mathrm{M}$ y $5 \mu \mathrm{l}$ de templado de ADN para un volumen final de $20 \mu \mathrm{L}$. Los parámetros de las reacciones para el equipo de RPC LightCycler 2.0 de Roche consistieron en un período inicial de activación de la enzima a $95^{\circ} \mathrm{C}$ por 10

Tabla 2. Detección de genes de virulencia cagA y vacA $m 1$ en pacientes con presencia de $H$. pylori en cavidad oral y mucosa gástrica

\begin{tabular}{|lcccc|} 
& \multicolumn{2}{c}{ Muestras orales } & \multicolumn{2}{c}{ Muestras gástricas } \\
& cagA & vacA m1 & cagA & vacA m1 \\
Grupo $1(n=1)$ & + & - & + & - \\
Grupo $2(n=7)$ & - & - & - & - \\
Grupo 3 $(n=2)$ & + & - & - & + \\
Grupo $4(n=1)$ & - & + & + & + \\
Grupo $5(n=1)$ & - & - & + & - \\
Grupo $6(n=1)$ & - & - & + & + \\
Grupo $7(n=5)$ & - & - & & - \\
\hline +: RPC en tiempo real positivo. -: RPC en tiempo real negativo. & \\
\hline
\end{tabular}

min, denaturación a $95^{\circ} \mathrm{C}$ por $10 \mathrm{~s}$, seguido de 50 ciclos de amplificación y alineamiento a $55^{\circ} \mathrm{C}$ por 10 s y elongación a $72^{\circ} \mathrm{C}$ por $8 \mathrm{~s}$. La presencia de productos de amplificación específicos para $H$. pylori y controles positivos de $H$. pylori ATCC 43504 fueron monitoreados por la detección fluorescente positiva a $530 \mathrm{~nm}$ de la sonda de hidrólisis FAM-TAMRA en el software del equipo y confirmado por los tamaños de producto esperado de 119 bp en gel de agarosa al 2\%, 80 v por 60 min en luz UV. Los controles positivos asociados al valor de Tm y amplicón, fueron previamente secuenciados en Macrogen Inc. Seoul, Korea.

Detección por RPC-TR con SYBR Green de los genes de virulencia de $H$. pylori cagA y vac ml y de las células totales con el gen $\beta$-actina como control interno. Se siguieron las instrucciones del fabricante de la mezcla lista para usar LightCycler ${ }^{\circledR}$ FastStart DNA Master PLUS SYBR Green I (catálogo 03515885001), la cual consistió en $10 \mu \mathrm{L}$ de $\mathrm{H}_{2} \mathrm{O}$ libre de nucleasas, $1,6 \mu 1$ de $\mathrm{MgCl}_{2} 25 \mathrm{mM}, 1,4 \mu \mathrm{L}$ de la mezcla de partidores cag $A$, vacA $\mathrm{ml}$ o $\beta$-actina respectivamente, de concentración stock $10 \mu \mathrm{M}, 2 \mu 1$ de mezcla de SYBR Green y $5 \mu 1$ de templado de ADN para un volumen final de $20 \mu \mathrm{L}$. Los parámetros para las reacciones de RPC LightCycler 2.0 sugeridos en el protocolo del fabricante consistieron en un período de activación de la enzima por $10 \mathrm{~min}$ a $95^{\circ} \mathrm{C}$, denaturación a $95^{\circ} \mathrm{C}$ por $10 \mathrm{~s}$, seguido por 50 ciclos de amplificación con una temperatura de alineamiento de $55^{\circ} \mathrm{C}$ por $10 \mathrm{~s}$ y elongación a $72^{\circ} \mathrm{C}$ por $14 \mathrm{~s}$, análisis de curva de melting $95^{\circ} \mathrm{C}, 69^{\circ} \mathrm{C}$ y $95^{\circ} \mathrm{C}$. La presencia de productos de amplificación específicos para $H$. pylori y controles positivos para $H$. pylori ATCC 43504 fueron monitoreados a $530 \mathrm{~nm}$ para el fluoróforo SYBR Green intercalado en la elongación a $72^{\circ} \mathrm{C}$ mediante el software del equipo, con el respectivo análisis de la curva de melting característica de producto único y luego confirmado por su tamaño 298 bp para cagA y 290 bp para $v a c A$ m1 y 289 bp para $\beta$-actina y asociación con la curva de melting en gel de agarosa al $2 \%, 80 \mathrm{v}$ por $60 \mathrm{~min}$ en transiluminador UV. Los controles positivos asociados al valor de Tm y amplicón, fueron previamente secuenciados en Macrogen Inc. Seoul, Korea.

\section{Resultados}

Como se observa en la Tabla 2, se obtuvo 7 combinaciones diferentes de genes $\operatorname{cag} A$ y $\operatorname{vac} A \mathrm{~m} 1$, las que denominamos grupos. Ocho pacientes, correspondientes a los grupos 1 y 2 , presentaban el mismo perfil de virulencia en estómago y boca, en base a los dos genes en estudio. Los restantes 10 pacientes, correspondientes a los grupos 3 al 7, presentaban cepas de $H$. pylori con perfiles genéticos asociados a virulencia diferentes en cavidad oral y mucosa gástrica.

También podemos observar en esta tabla que no se 
detectó en paciente alguno la presencia simultánea de H. pylori con su genotipo más virulento $(\operatorname{cag} A+, v a c A$ $\mathrm{m} 1+$ ), ni en cavidad oral ni en mucosa gástrica. Sin embargo, al analizar por separado ambos compartimentos, se observa que en las muestras gástricas, dos pacientes (correspondientes a los grupos 4 y 5) poseían este genotipo. Dos pacientes presentaban cepas de $H$. pylori con el perfil $\operatorname{cag} A+, v a c A$ m1- y cinco el perfil $\operatorname{cag} A$-, vacA $\mathrm{m} 1+$. Por otra parte, en las muestras orales, tres pacientes presentaban el genotipo asociado a virulencia $\operatorname{cag} A+, v a c A$ m1- (correspondientes a los grupos 1 y 3 ), un paciente presentaba el genotipo de $H$. pylori cagA-, vacA $\mathrm{m} 1+\mathrm{y}$ los restantes pacientes $(\mathrm{n}=14)$ presentaban cepas de $H$. pylori con el genotipo $\operatorname{cag} A-$, vacA $\mathrm{m1}$-.

\section{Discusión}

Varios estudios han demostrado la presencia de $H$. pylori simultáneamente en cavidad oral y mucosa gástrica, mostrando diferentes prevalencias, dependiendo de la población estudiada, la selección de la muestra y técnicas de detección de la bacteria ${ }^{26-33,40}$. Mapstone ${ }^{41}$, demostró que la presencia de $H$. pylori en la boca de pacientes dispépticos es importante para su diseminación y considera la cavidad oral una potencial fuente de reinfección luego de la erradicación del microorganismo del estómago. Esto último, fue una de las motivaciones para realizar esta investigación.

Sin embargo, es sabido que la identificación de especies bacterianas de la cavidad oral es particularmente difícil debido a que existen más de 500 especies en la placa bacteriana oral ${ }^{42}$ y más de 700 especies en la cavidad oral en general $^{43}$. Por ello, es necesario contar con métodos lo más específicos y sensibles para la detección e identificación de H. pylori. La especificidad en la identificación de este microorganismo se evidencia en su cultivo bacteriológico, y se ha observado que muestras con cultivos gástricos positivos para $H$. pylori también presentan resultados positivos para muestras orales mediante RPC-TR ${ }^{34}$, avalando la especificidad de este método molecular para esta bacteria. Sin embargo, en cuanto a la sensibilidad, algunos autores han reportado esfuerzos insatisfactorios para cultivar H. pylori de la cavidad oral ${ }^{44}$. Esto puede deberse a un bajo número de bacteria en la boca debido a una inhibición producida por la compleja microbiota oral o debido a la forma cocoide de la bacteria, que no es detectable mediante técnicas de cultivo tradicionales ${ }^{45}$. La conversión de la forma bacilar a cocoide se acompaña de una gran disminución de las unidades formadoras de colonias por mililitro $(\mathrm{ufc} / \mathrm{ml})^{46}$. Se sabe que la forma cocoide es una forma viable pero no cultivable en los métodos clásicos de cultivo de este microorganismo, como el agar Columbia ${ }^{47,48}$. Recientemente el trabajo de Al-Sulami y cols. ${ }^{49}$, presenta la primera evidencia de crecimiento de $H$. pylori en agua potable municipal, esto gracias al desarrollo de un medio selectivo, llamado agar modificado urea Columbia (MCUA) $)^{50}$.

Diversos autores ${ }^{51,52}$ discuten la importancia de desarrollar nuevas técnicas para la detección, aislamiento y cultivo de bajos recuentos de H. pylori en placa bacteriana oral. De acuerdo a nuestros resultados, creemos que este problema ha sido parcialmente superado, pues es posible detectar el ADN de la bacteria, aunque no se logre su aislamiento. Sugerimos, por tanto, que para las muestras orales con bajos números de $H$. pylori, la técnica de elección para su detección sea la RPC-TR.

Si bien cabe destacar que en este trabajo se incluyeron sólo dos genes asociados a virulencia, los genes seleccionados, $\operatorname{cag} A$ y vacA $\mathrm{m} 1$, partes del cromosoma bacteriano, siguen siendo importantes marcadores genéticos asociados a virulencia en $H$. pylori ${ }^{12}$. Específicamente $\operatorname{cag} A$ se encuentra formando parte de las islas de patogenicidad cag (cagPAI $)^{12}$. Las islas de patogenicidad en bacterias gramnegativas, como lo es $H$. pylori, suelen poseer genes para movilizarse, por lo tanto, es posible que provengan de un proceso de captación de información genética tipo conjugación y tener capacidad de transferirse. No sucede lo mismo con el gen vacA, de localización cromosomal, pero no formando parte de una isla ${ }^{53}$.

Además, trabajos previos de nuestro grupo muestran la importancia de estos genes de $H$. pylori en población chilena, pues se encuentran asociados a patologías gastro-duodenales ${ }^{18,19}$. Con respecto al bajo al número de pacientes incluidos, cabe señalar que no es fácil contar con muestras obtenidas simultáneamente de cavidad oral y mucosa gástrica de un mismo paciente; no obstante se reconoce esta limitación.

Al analizar los resultados se observa que 14 de 18 pacientes presentaban en su cavidad oral cepas de $H$. pylori con el genotipo cagA-, vacAm1-, lo que sugiere que la mayoría de los pacientes son colonizados oralmente por los genotipos menos virulentos de H. pylori. A su vez, en el estómago, la mitad de los pacientes estaban colonizados por este genotipo de $H$. pylori. Por lo tanto, podemos pensar que efectivamente los pacientes incluidos en este estudio, en su mayoría poseían cepas con genotipos asociados a menor virulencia.

Por otra parte, los resultados sugieren que en los ocho pacientes, en los cuales se detectaron los mismos genotipos de virulencia en las muestras orales y gástricas, se trate de las mismas cepas de H. pylori. Sin embargo, para asegurar esto se deberían secuenciar los genomas completos de las cepas detectadas, ya que esta es la única forma de demostrar identidad genética.

En otros ocho pacientes (grupos 4 al 7) se detectaron perfiles de cepas menos virulentas en la cavidad oral respecto del estómago, en estos casos se puede pensar que se trata de cepas diferentes. Sin embargo, también es probable que las cepas de $H$. pylori detectadas en cavidad 
oral fuesen las mismas que detectamos en las muestras gástricas para estos pacientes. Esto podría ser explicado por una posible recombinación genética, ya fuese en la misma cavidad oral o en el estómago, haciéndose más virulentas. Lo anterior, puede ser avalado por un estudio reciente de Kennemann y cols. ${ }^{54}$, quienes señalan que el genoma de $H$. pylori evoluciona durante la infección humana y que es estable en ausencia de una infección mixta. En el presente trabajo no se investigó la existencia o no de infección mixta; sin embargo, trabajos previos sí han señalado la existencia de infección mixta en pacientes chilenos $^{18,19}$. Sólo en dos pacientes (grupo 3) se observó un perfil más virulento en la cavidad oral en relación al estómago. En estos casos lo más probable es que se trate de cepas diferentes. Esto se basa en que se sabe que estos genes están en el cromosoma y no en material de ADN extra-cromosómico, como los plásmidos, donde sus genes son más fáciles de perder, por ejemplo por curación o autocuración plasmídica (pérdida natural del plásmido). Sin embargo, dada la evolución del genoma bacteriano en el organismo humano, no es posible descartar totalmente que puedan ser las mismas cepas que en el seno del estómago perdieron su gen $\operatorname{cag} A^{54}$. Nuevamente, para dilucidar esto se deberían secuenciar los genomas completos de las cepas de $H$. pylori detectadas.

Los resultados de la presente investigación, en su conjunto, muestran que existe una variedad de genotipos de virulencia en $H$. pylori, basados en los genes cagA y $v a c A \mathrm{~m} 1$ en el estómago y la cavidad oral, predominando en los pacientes incluidos en este estudio las cepas con genotipos asociados a menor virulencia (cagA-, vacA $\mathrm{m} 1-$ ). Conociendo el potencial de virulencia de la bacteria podemos deducir algo sobre el pronóstico, pues los indi- viduos colonizados por cepas $\operatorname{cag} A, \operatorname{vac} A \mathrm{~m} 1$ negativas podrían corresponder a la mayoría de la población que está colonizada por la bacteria, pero son asintómaticos durante toda su vida. En cambio, los individuos colonizados por cepas $\operatorname{cag} A$, vacA $\mathrm{m} 1$ positivas, son los que en su mayoría llegan a ser sintomáticos ${ }^{12}$.

Se sugieren futuros estudios con mayor cantidad de pacientes e incluyendo otros genes asociados a virulencia, como vacA s1 y dupA; estos últimos porque en estudios previos de nuestro grupo (resultados proyecto Fondef D03i1109 aún no publicados) también han demostrado ser importantes para la población chilena.

\section{Resumen}

Objetivo: Comparar el genotipo de virulencia (genes cagA y vacA m1) de Helicobacter pylori, obtenido simultáneamente de mucosa gástrica y cavidad oral. Material y Métodos: Para esto se incluyeron muestras de biopsias gástricas de 18 pacientes. Las muestras orales de estos pacientes fueron obtenidas de placa bacteriana y saliva del piso de boca y base de la lengua. Las muestras fueron estudiadas con RPC convencional y RPC en tiempo real (RPC-TR). Los genes de virulencia cagA y vacA $\mathrm{m} 1$ fueron estudiados con RPC-TR. Resultados: De acuerdo a la presencia o ausencia de los genes de virulencia cag $A$ y vacA $\mathrm{m} 1$ detectados en las muestras gástricas y orales, se pudieron diferenciar siete combinaciones diferentes. Conclusión: Estos resultados sugieren que existe una variedad de genotipos de virulencia en Helicobacter pylori en el estómago y la cavidad oral, predominando en los pacientes incluidos en este estudio las cepas con genotipos asociados a menor virulencia ( $\operatorname{cag} A$-, $\operatorname{vac} A \mathrm{ml}-$ ).

\section{Referencias bibliográficas}

1.- Ferreccio C, Rollán A, Harris P R, Serrano C, Gederlini A, Margozzini P, et al. Gastric cancer is related to early Helicobacter pylori infection in a high-prevalence country. Cancer Epidemiol Biomarkers Prev 2007; 16: 662-7.

2.- Ministerio de Salud de Chile. Estadísticas de natalidad y mortalidad. url: http://www.minsal.cl.

3.- Prado V. Emergent infectious diseases: a new problem? Rev Med Chile 1996; 124: 7-10.

4.- Araya J C, Anabalón L, Roa I, Bravo M, Villaseca M A, Guzmán P, et al. Association between Helicobacter pylori genotype and the severity of gastritis in infected adults. Rev Med Chile 2004; 132: 1345-54.

5.- González F C G, Serrano H C, Harris P R. Diagnosis of Helicobacter pylori infection in children based on stool antigen test. Rev Med Chile 2007; 135: 182-8.

6.- Luo Y H, Yan J, Mao Y F. Helicobacter pylori lipopolysaccharide: biological activities in vitro and in vivo, pathological correlation to human chronic gastritis and peptic ulcer. World J Gastroenterol 2004; 10: 2055-9.

7.- Salgado F, García A, Oñate A, González C, Kawaguchi F. Increased in-vitro and in-vivo biological activity of lipopolisaccharide extracted from clinical low virulence vacA genotype Helicobacter pylori strains. J Med Microbiol 2002; 51: 771-6.

8.- Atherton J C. The pathogenesis of Helicobacter pylori-induced gastroduodenal diseases. Annu Rev Pathol 2006; 1: 63-96.

9.- Peek R M Jr, Crabtree J. Helicobacter infection and gastric neoplasia. J Pathol 2006; 208: 233-48.

10.- Willhite D C, Cover T L, Blanke S R. Cellular vacuolation and mitochondrial cytochrome c release are independent outcomes of Helicobacter pylori vacuolating cytotoxin activity that are each dependent on membrane channel formation. J Biol Chem 2003; 278: 48204-9
11.- Atherton J C, Peek R M Jr, Tham K T, Cover T L, Blaser M J. Clinical and pathological importance of heterogeneity in $v a c A$, the vacuolating cytotoxin gene of Helicobacter pylori. Gastroenterology 1997; 112: 92-9.

12.- González C A, Figueiredo C, Lic C B, Ferreira R M, Pardo M L, Ruiz Liso J M, et al. Helicobacter pylori cagA and vacA genotypes as predictors of progression of gastric preneoplastic lesions: A long-term follow-up in a high risk area in Spain. Am J Gastroenterol 2011; 106: 867-74.

13.- Blaser M J, Berg DE. Helicobacter pylori diversity and risk of human disease. J Clin Invest 2001; 107: 767-73.

14.- Atherton J C, Cao P, Peek RM Jr, Tummuru M K, Blaser M J, Cover T L. Mosaicism in vacuolating cytotoxin alleles of Helicobacter pylori. Association of specific vacA types with cytotoxin production and peptic ulceration. J Biol Chem 1995; 270: 17771-7. 
15.- Van Doorn L J, Figueiredo C, Sanna R, Pena A S, Midolo P, Atherton J C, et al. Expanding allelic diversity of Helicobacter pylori vacA. J Clin Microbiol 1998; 36: 2597- 603.

16.- Tomb J F, White O, Kerlavagh A R, Clayton R A, Sutton C C, Fleischmann R D, et al. The complete genome sequence of the gastric pathogen Helicobacter pylori. Nature 1997; 388: 539-47.

17.- Covacci A, Telford J L, Del Giudice G, Parsonnet J, Rappuoli R. Helicobacter pylori virulence and genetic geography. Science 1999; 284: 1328-33.

18.- Martínez A, González C, Kawaguchi F, Montoya R, Corvalán A, Madariaga J, et al. Helicobacter pylori: cagA analysis and vacA genotyping in Chile. Detection of a $s 2 / \mathrm{m} 1$ strain. Rev Med Chile 2001; 129: 1147-53.

19.- García A, Barra R, Delgado C, Kawaguchi F, Trabal N, Montenegro S, et al. Genotyping of clinical isolates of Helicobacter pylori by cagA, $v a c A$ and babA2 virulence associated genes. First detection of a babA2 positive strain in Chilean patients. Rev Med Chile 2006; 134: 981-8.

20.- Ozdemir A, Mas M R, Sahin S, Saglamkaya U, Ateskan U. Detection of Helicobacter pylori colonization in dental plaques and tongue scrapings of patients with chronic gastritis. Quintessence Int 2001; 32: 131-4.

21.- Song Q, Haller B, Schmid RM, Adler G, Bode G. Helicobacter pylori in dental plaque: a comparison of different PCR primer sets. Dig Dis Sci 1999; 44: 479-84.

22.- Majmudar P, Shah S M, Dhunjibhoy K R, Desai H G. Isolation of Helicobacter pylori from dental plaques in healthy volunteers. Indian J Gastroenterol 1990; 9: 271-2.

23.- Kim N, Lim S H, Lee K H, You J Y, Kim J M, Lee N R, et al. Helicobacter pylori in dental plaque and saliva. Korean J Intern Med 2000; 15: 187-94.

24.- Song Q, Spahr A, Schmid RM, Adler G, Bode G. Helicobacter pylori in the oral cavity: high prevalence and great DNA diversity. Dig Dis Sci 2000; 45: 2162-7.

25.- Desai H G, Gill H H, Shankaran K, Mehta P R, Prabhu S R. Dental plaque: a permanent reservoir of Helicobacter pylori? Scand J Gastroenterol 1991; 26: 1205-8.

26.- Bürgers R, Schneider-Brachert W, Reischl U, Behr A, Hiller K A, Lehn N, et al. Helicobacter pylori in human oral cavity and stomach. Eur $\mathrm{J}$ Oral Sci 2008; 116: 297-304.

27.- Wang J, Chi D S, Laffan J J, Li C, Ferguson D A Jr, Litchfield P, et al. Comparison of cytotoxin genotypes of Helicobacter pylori in stomach and saliva. Dig Dis Sci 2002; 47: 1850-6.

28.- $\mathrm{Hu} \mathrm{W}$, Cao C, Meng H, Zhang J, Ma D, Zhang L. Detection and analysis of Helicobacter pylori in oral cavity and stomach from chronic gastritis patients. Zhonghua Yi Xue Za Zhi 2002; 82: 1037-41.

29.- Allaker R P, Young K A, Hardie J M, Domizio P, Meadows N J. Prevalence of Helicobacter pylori at oral and gastrointestinal sites in children: evidence for possible oral-tooral transmission. J Med Microbiol 2002; 51: 312-27.

30.- Karczewska E, Konturek J E, Konturek P C, Czesnikiewicz M, Sito E, Bielanski W, et al. Oral cavity as a potential source of gastric reinfection by Helicobacter pylori. Dig Dis Sci 2002; 47: 978-86.

31.- Cheng L H, Webberley M, Evans M, Hanson N, Brown R. Helicobacter pylori in dental plaque and gastric mucosa. Oral Surg Oral Med Oral Pathol Oral Radiol Endod 1996; 81: 421-3.

32.- Silva D G, Tinoco E M, Rocha G A, Guerra J B, Saraiva I E, Queiroz D M. Helicobacter pylori transiently in the mouth may participate in the transmission of infection. Mem Inst Oswaldo Cruz 2010; 105: 657-60.

33.- Zou Q H, Li R Q. Helicobacter pylori in oral cavity and gastric mucosa: a meta-analysis. J Oral Pathol Med 2011; 40: 317-24.

34.- Sepúlveda E, Briceño C, Spencer M L, Quilodrán S, Brethauer U, Moreno J, et al. Detección de Helicobacter pylori en mucosa gástrica y cavidad oral. Gastroenterol Latinoamer 2008; 19: 73-9.

35.- Assumpcão M B, Martins L C, Melo Barbosa H P, Barile K A, de Almeida S S, Assumpção P P, et al. Helicobacter pylori in dental plaque and stomach of patients from Northern Brazil. World J Gastroenterol 2010; 16: 3033-9.

36.- Silva Rossi-Aguiar VP, Navarro-Rodríguez T, Mattar R, Siqueira de Melo Peres M P, Correa Barbuti R, Silva F M, et al. Oral cavity is not a reservoir for Helicobacter pylori in infected patients with functional dyspepsia. Oral Microbiol Immunol 2009; 24: 255-9.

37.- Yamazaki S, Kato S, Matsukura N, Ohtani M, Ito $\mathrm{Y}$, Suto $\mathrm{H}$, et al. Identification of Helicobacter pylori and cagA genotype in gastric biopsies using highly sensitive real-time PCR as a new diagnostic tool. FEMS Immunol Med Microbiol 2005; 44: 261-8.

38.- Ruzsovics A, Molnar B, Unger Z, Tulassay Z, Pronai L. Determination of Helicobacter pylori $\operatorname{cag} A, v a c A$ genotypes with real-time PCR melting curve analysis. J Physiol Paris 2001; 95: 369-77.

39.- Yamazaki S, Yamakawa A, Okuda T, Ohtani M, Suto H, Ito Y, et al. Distinct diversity of vacA, cagA, and cage genes of Helicobacter pylori associated with peptic ulcer in japan. J Clin Microbiol 2005; 43: 3906-16.

40.- Song Q, Lange T, Spahr A, Adler G, Bode G. Characteristic distribution pattern of Helicobacter pylori in dental plaque and saliva detected with nested PCR. J Med Microbiol 2000; 49: 349-53
41.- Mapstone N P, Lynch D A, Lewis F A, Axon A T, Tompkins D S, Dixon M F, et al. Identification of Helicobacter pylori DNA in the mouths and stomachs of patients with gastritis using PCR. J Clin Pathol 1993; 46: 540-3.

42.- Kroes I, Lepp P W, Relman D A. Bacterial diversity within the human subgingival crevice. Proc Natl Acad Sci USA 1999; 96: 14547-52.

43.- Aas J A, Paster B J, Stokes L N, Olsen I, Dewhirst F E. Defining the normal bacterial flora of the oral cavity. J Clin Microbiol 2005; 43: 5721-32.

44.- Dowsett S A, Kowolik M J. Oral Helicobacter pylori: can we stomach it? Crit Rev Oral Biol Med 2003; 14: 226-33.

45.- Bode G, Mauch F, Malfertheiner P. The coccoid forms of Helicobacter pylori. Criteria for their viability. Epidemiol Infect 1993; 111: 483-90

46.- Catrenich C E, Makin K M. Characterization of the morphologic conversion of Helicobacter pylori from bacillary to coccoid forms. Scand J Gastroenterol Suppl 1991; 181: 58-64.

47.- Hua J, Ho B. Is the coccoid form of Helicobacter pylori viable? Microbios 1996; 87: 103-12.

48.- Can F, Karahan C, Dolapci I, Demirbilek M, Tekeli A, Arslan H. Urease activity and urea gene sequencing of coccoid forms of Helicobacter pylori induced by different factors. Curr Microbiol 2008; 56: 150-5.

49.- Al-Sulami A A, Al-Taee A M, Juma'a M G. Isolation and identification of Helicobacter pylori from drinking water in Basra governorate, Iraq. East Mediterr Health J 2010; 16: 920-5.

50.- Al-Sulami A, Al-Kiat HS, Bakker L K, Hunoon H. Primary isolation and detection of Helicobacter pylori from dyspeptic patients: a simple, rapid method. East Mediterr Health J 2008; 14: 268-76.

51.- Siavoshi F, Salmanian A H, Akbari F, Malekzadeh R, Massarat S. Detection of Helicobacter pylori-specific genes in the oral yeast. Helicobacter 2005; 10 : 318-22.

52.- Salmanian A H, Siavoshi F, Akbari F, Afshari A, Malekzadeh R. Yeast of the oral cavity is the reservoir of Helicobacter pylori. J Oral Pathol Med 2008; 37: 324-8.

53.- Censini S, Lange C, Xiang Z, Crabtree J E, Ghiara P, Borodovsky M, et al. cagA pathogenecity island of Helicobacter pylori, encodes type I-specific and disease-associated virulence factors. Proc Natl Acad Sci USA 1996; 93: 14648-53.

54.- Kennemann L, Didelot X, Aebischer T, Kuhn S, Drescher B, Droege M, et al. Helicobacter pylori genome evolution during human infection. Proc Natl Acad Sci USA 2011; 108: 5033-8. 